\title{
Evolution of the core and pan-genome of Streptococcus: positive selection, recombination, and genome composition Tristan Lefébure and Michael J Stanhope
}

Address: Department of Population Medicine and Diagnostic Sciences, College of Veterinary Medicine, Cornell University, Ithaca, NY 14853, USA.

Correspondence: Michael J Stanhope. Email: mjs297@cornell.edu

Published: 2 May 2007

Genome Biology 2007, 8:R7I (doi:I0.I I86/gb-2007-8-5-r7I)

The electronic version of this article is the complete one and can be found online at http://genomebiology.com/2007/8/5/R71
Received: 28 November 2006

Revised: 24 April 2007

Accepted: 2 May 2007

(C) 2007 Lefébure and Stanhope; licensee BioMed Central Ltd.

This is an open access article distributed under the terms of the Creative Commons Attribution License (http://creativecommons.org/licenses/by/2.0), which permits unrestricted use, distribution, and reproduction in any medium, provided the original work is properly cited.

\begin{abstract}
Background: The genus Streptococcus is one of the most diverse and important human and agricultural pathogens. This study employs comparative evolutionary analyses of 26 Streptococcus genomes to yield an improved understanding of the relative roles of recombination and positive selection in pathogen adaptation to their hosts.

Results: Streptococcus genomes exhibit extreme levels of evolutionary plasticity, with high levels of gene gain and loss during species and strain evolution. S. agalactiae has a large pan-genome, with little recombination in its core-genome, while $S$. pyogenes has a smaller pan-genome and much more recombination of its core-genome, perhaps reflecting the greater habitat, and gene pool, diversity for $S$. agalactiae compared to $S$. pyogenes. Core-genome recombination was evident in all lineages ( $18 \%$ to $37 \%$ of the core-genome judged to be recombinant), while positive selection was mainly observed during species differentiation (from II\% to $34 \%$ of the core-genome). Positive selection pressure was unevenly distributed across lineages and biochemical main role categories. S. suis was the lineage with the greatest level of positive selection pressure, the largest number of unique loci selected, and the largest amount of gene gain and loss.
\end{abstract}

Conclusion: Recombination is an important evolutionary force in shaping Streptococcus genomes, not only in the acquisition of significant portions of the genome as lineage specific loci, but also in facilitating rapid evolution of the core-genome. Positive selection, although undoubtedly a slower process, has nonetheless played an important role in adaptation of the core-genome of different Streptococcus species to different hosts.

\section{Background}

Microbial pathogens show surprising capacity for adaptation to new hosts, antibiotics, or immune systems. Three principal mechanisms are regarded as important in this adaptive potential: Darwinian, or positive selection, favoring the fixation of advantageous mutations; acquisition of new genetic material by lateral DNA exchange (that is, recombination); and gene regulation. Several studies have suggested that recombination might be the key factor in adaptation of pathogens and that the recombination rates of bacteria might be higher than their mutation rates [1-4]. At the same time, there is a portion of the genome - the core-genome - that is thought 
to be representative of bacterial taxa, at various taxonomic levels [5]. Recent molecular evolution analyses of Escherichia coli and Salmonella enterica [6,7] have identified genes under positive selection pressure in the core-genome of these enteric bacteria. Genome sequence data are now available for numerous species of several genera of bacteria, providing the possibility of using comparative evolutionary genomic approaches to assess positive selection pressure and the role of horizontal gene transfer in the evolution of the coregenome of a bacterial genus.

One such important bacterial genus is Streptococcus, which includes some of the most important human and agricultural pathogens, causing a wide range of different diseases, and inflicting significant morbidity and mortality throughout the world, as well as resulting in significant economic burden. Twenty six genomes of Streptococcus are available on public databases belonging to six different species, including $S$. pneumoniae, S. agalactiae, S. pyogenes, $S$. thermophilus, $S$. mutans and S. suis. S. pyogenes (Group A Streptococcus; GAS), is responsible for a wide range of human diseases, including pharyngitis, impetigo, puerperal sepsis, necrotizing fasciitis ('flesh-eating disease'), scarlet fever, the postinfection sequelae glomerulonephritis and rheumatic fever. In addition, S. pyogenes has recently been associated with Tourette's syndrome and movement and attention deficit disorders [8]. A resurgence of $S$. pyogenes infections has been observed since the mid-1980s. S. agalactiae is another important human pathogen and is the leading cause of bacterial sepsis, pneumonia, and meningitis in US and European neonates [9]. Although S. agalactiae normally behaves as a commensal organism that colonizes the genital or gastrointestinal tract of healthy adults, it can cause life threatening invasive infection in susceptible hosts, such as newborns, pregnant women, and nonpregnant adults with chronic illnesses [10]. S. agalactiae was first recognized as a pathogen in bovine mastitis [11]. S. pneumoniae is the leading cause of human bacterial infection worldwide [12], although paradoxically, is primarily carried asymptomatically. It has been an object of medical study and scrutiny for over a century. $S$. mutans is implicated as the principal causative agent of human dental caries (tooth decay) [13]. S. thermophilus is a non-pathogenic, food microorganism, widely used in the dairy product industry. S. suis is responsible for a variety of diseases in pigs, including meningitis, septicemia, arthritis, and pneumonia [14]. It is also a zoonotic pathogen that causes occasional cases of meningitis and sepsis in humans, but has recently also been implicated in outbreaks of streptococcal toxic shock syndrome [15].

A recent comparative genomic analysis of five of these above mentioned streptococcal species (S. suis not included), focused on understanding the role of lateral gene transfer in shaping the genomes of each of these lineages, and analyzed some of the species specific genes for potential adaptive evolution [16]. Species or strain specific loci are often the focus of attempts to understand adaptive differences in bacteria However, with the exception of the Chen et al. [7] study on $E$. coli, assessments of adaptive evolution in the core-genome components of other bacterial species have not been thoroughly explored. In addition to individual genome sequences for several species of Streptococcus, there are also complete genome sequences available for multiple strains of $S$. agalactiae, S. pyogenes, and S. thermophilus. Genome wide molecular selection analyses, designed to assess selection pressure across the entire core-genome of different species and strains of Streptococcus have not been reported, and also no published reports have attempted to address the relative role of selection versus recombination in the diversification of the core-genome of Streptococcus.

Along with the burgeoning increase in microbial genome sequence data there has been a concomitant development of sophisticated methods for detecting positive selection in protein coding genes. These methods can be used to compare orthologous DNA sequence data across the entire genomes of the available species within the genus Streptococcus. Ziheng Yang, Rasmus Nielsen and colleagues [17-21] have developed powerful statistical methods for detecting adaptive molecular evolution. Their methods compare synonymous and nonsynonymous substitution rates in protein coding genes and regard a nonsynonymous rate elevated above the synonymous rate as evidence for positive or Darwinian selection. Positive natural selection leads to the fixation of advantageous mutations driven by natural selection, and is the fundamental process behind adaptive changes in genes and genomes, leading to evolutionary innovations and species differences. A significant advancement on many earlier methods, which averaged over sites and time, their methods are designed to detect positive selection at individual sites and lineages [20]. Our study employs these powerful selection methods to assess positive selection pressure across the coregenome components of the genus Streptococcus, as well as several species of Streptococcus, while concomitantly assessing levels of recombination within the core-genome.

Concomitant with the identification of bacterial coregenomes, it has become evident that there is an apparently dispensable portion of bacterial genomes, consisting of partially shared and strain-specific genes that can, even within a particular species, represent a surprisingly large proportion (for example, [22]). The concept of dispensable portions of genomes implies that genes have been lost and gained since separation from common ancestors, which in turn implies that this loss and gain can be estimated from reconstructed genome composition. This sort of approach has been undertaken previously, including for a few species of Streptococcus [23], with one of the resulting conclusions being that gene gain tends to be much greater than gene loss. An additional purpose of this paper is to compare gene gain and loss within and between Streptococcus species, making use of the larger comparative data set of species and strains now available, and 
Table I

\begin{tabular}{|c|c|c|c|c|c|c|}
\hline \multicolumn{7}{|c|}{ Genomes analyzed } \\
\hline Species & Strain & Refseq accession number & Status & CDS & Serotype & References \\
\hline S. pyogenes & MGASI0270 & GenBank:NC 008022 & Complete & I,987 & M2 & [46] \\
\hline S. pyogenes & MGASI0750 & GenBank:NC 008024 & Complete & 1,979 & M4 & [46] \\
\hline S. pyogenes & MGAS2096 & GenBank:NC 008023 & Complete & I,898 & $\mathrm{MI} 2$ & [46] \\
\hline S. pyogenes & MGAS9429 & GenBank:NC 008021 & Complete & $\mathrm{I}, 877$ & $M 12$ & {$[46]$} \\
\hline S. pyogenes & MI GAS & GenBank:NC 002737 & Complete & I,697 & $\mathrm{MI}$ & [76] \\
\hline S. pyogenes & MGAS5005 & GenBank:NC 007297 & Complete & 1,865 & MI & [77] \\
\hline S. pyogenes & MGAS8232 & GenBank:NC 003485 & Complete & $\mathrm{I}, 845$ & MI8 & {$[78,79]$} \\
\hline S. pyogenes & MGAS6I80 & GenBank:NC 007296 & Complete & I,894 & $M 28$ & {$[80]$} \\
\hline S. pyogenes & MGAS3I5 & GenBank:NC 004070 & Complete & I,865 & M3 & [79] \\
\hline S. pyogenes & SSI-I & GenBank:NC 004606 & Complete & $|, 86|$ & M3 & {$[81]$} \\
\hline S. pyogenes & MGASI0394 & GenBank:NC 006086 & Complete & I,886 & M6 & {$[82]$} \\
\hline S. pneumoniae & R6 & GenBank:NC 003098 & Complete & 2,043 & & [83] \\
\hline S. pneumoniae & TIGR4 & GenBank:NC_003028 & Complete & 2,094 & & {$[84]$} \\
\hline S. mutans & UAI59 & GenBank:NC 004350 & Complete & 1,960 & & {$[85]$} \\
\hline S. agalactiae & $2603 V / R$ & GenBank:NC 004116 & Complete & 2,124 & & [86] \\
\hline S. agalactiae & A909 & GenBank:NC 007432 & Complete & 1,996 & & {$[22]$} \\
\hline S. agalactiae & NEM3I6 & GenBank:NC_004368 & Complete & 2,094 & & [9] \\
\hline S. agalactiae & 515 & GenBank:NZ AAJP00000000 & WGS & 2,275 & & {$[22]$} \\
\hline S. agalactiae & $\mathrm{CJB} I I I$ & GenBank:NZ AAJQ00000000 & WGS & 2,197 & & [22] \\
\hline S. agalactiae & $\mathrm{COHI}$ & GenBank:NZ AA/R00000000 & WGS & 2,376 & & [22] \\
\hline S. agalactiae & $\mathrm{H} 36 \mathrm{~B}$ & GenBank:NZ AAJS00000000 & WGS & 2,376 & & {$[22]$} \\
\hline S. agalactiae & |8RS2I & GenBank:NZ AAJO00000000 & WGS & 2,146 & & {$[22]$} \\
\hline S. suis & $89 / 1591$ & GenBank:NZ AAFA00000000 & WGS & 1,896 & & \\
\hline S. thermophilus & CNRZI066 & GenBank:NC 006449 & Complete & 1,915 & & [87] \\
\hline S. thermophilus & LMG I83II & GenBank:NC 006448 & Complete & 1,889 & & {$[87]$} \\
\hline S. thermophilus & LMD-9 & GenBank:NZ AAGS00000000 & WGS & I,835 & & \\
\hline
\end{tabular}

CDS, number of protein coding sequences; WGS, whole genome shotgun.

to compare that history with histories of positive selection and recombination in the core-genome.

\section{Results \\ Pan-genome, core-genome, and evolution of genome composition}

The number of protein coding genes per genome within the various strains and species of Streptococcus is relatively similar (ranging from 1,697 to 2,376; Table 1), but the gene composition of these genomes is much more variable. Based on the gene content table obtained by OrthoMCL (Additional data file 1), three strains of $S$. agalactiae, $S$. pyogenes or $S$. thermophilus share about $75 \%$ of their genes, and three different species of Streptococcus share only around half of their genes (Figure 1). This latter result appears to be independent of the particular strains or species involved in the comparison and of their phylogenetic affinities. Even with the inclusion of 26 genomes, the total number of possible genes - the pangenome - of Streptococcus appears not to have been reached, as depicted in the gene accumulation curve (Figure 2), and we estimate the Streptococcus pan-genome probably surpasses 6,000 genes. A surprising $21 \%$ of the genes in the pangenome of the genus Streptococcus (based on these 26 genome sequences), were represented in only one lineage, suggesting a remarkable degree of lateral gene transfer in shaping the genomes of each of these taxa (Figure 3). Within species, the pan-genome size also remains uncertain, although our estimates suggest that the pan-genome size of $S$. pyogenes is smaller, and better estimated with the currently available data, than that of $S$. agalactiae (Figure 2).

In contrast to the pan-genome estimates, the number of genes in common between the different species within the genus Streptococcus - the core-genome - appears to reach a plateau around 600 genes (Figures 2 and 3). Next to the genome specific genes and the genes shared by only two genomes, the genes of the core-genome were the third most common genes (11\%; Figure 3), suggesting they form a coherent group. Similarly, the estimated core-genome for $S$. pyogenes, based on the 11 available strains, plateaus around 1,400 genes. The pattern was less clear for $S$. agalactiae, where the estimate of 


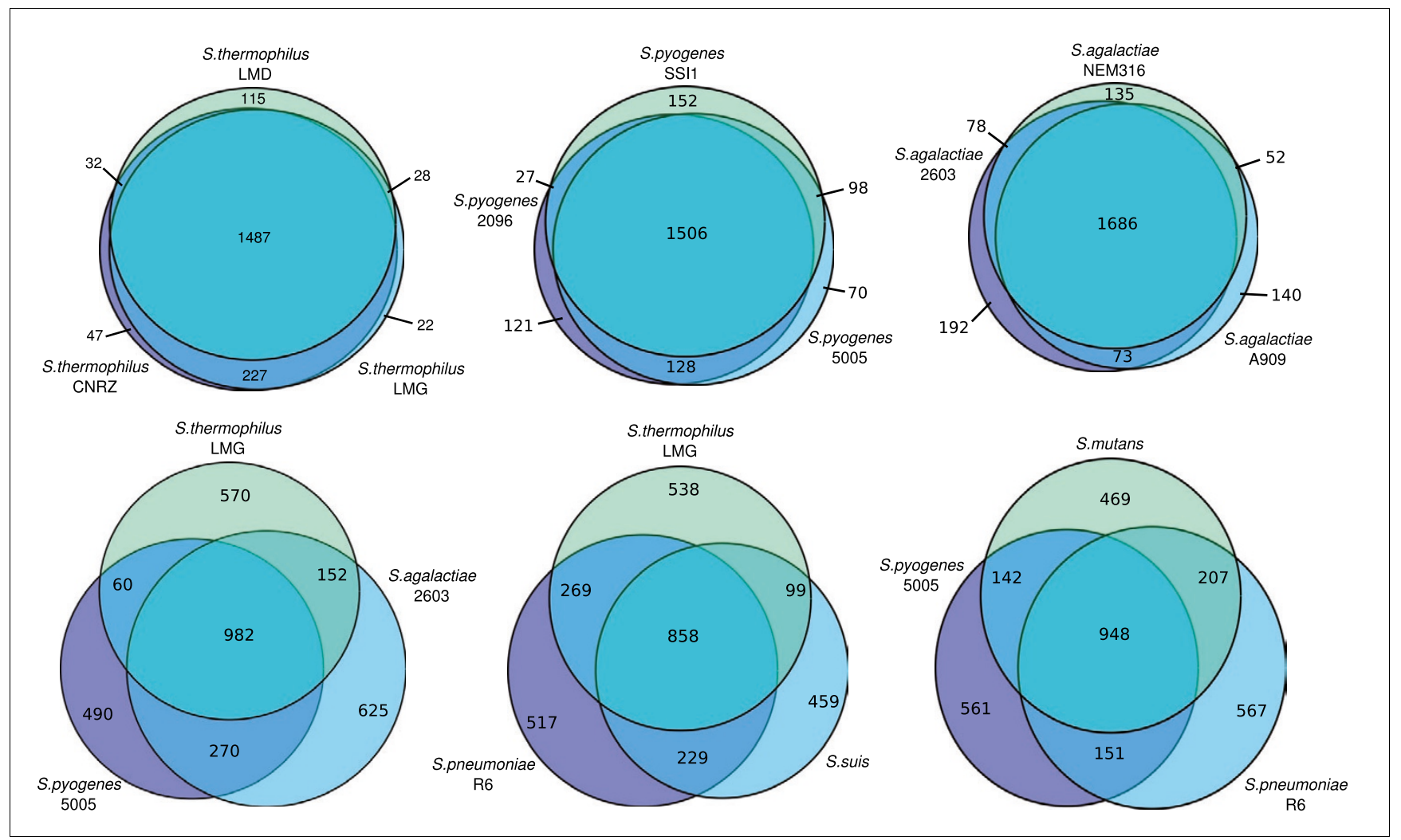

Figure I

Venn diagram for six sets of three taxa. Above are taxa of the same species and below are taxa of different species. The surfaces are approximately proportional to the number of genes.

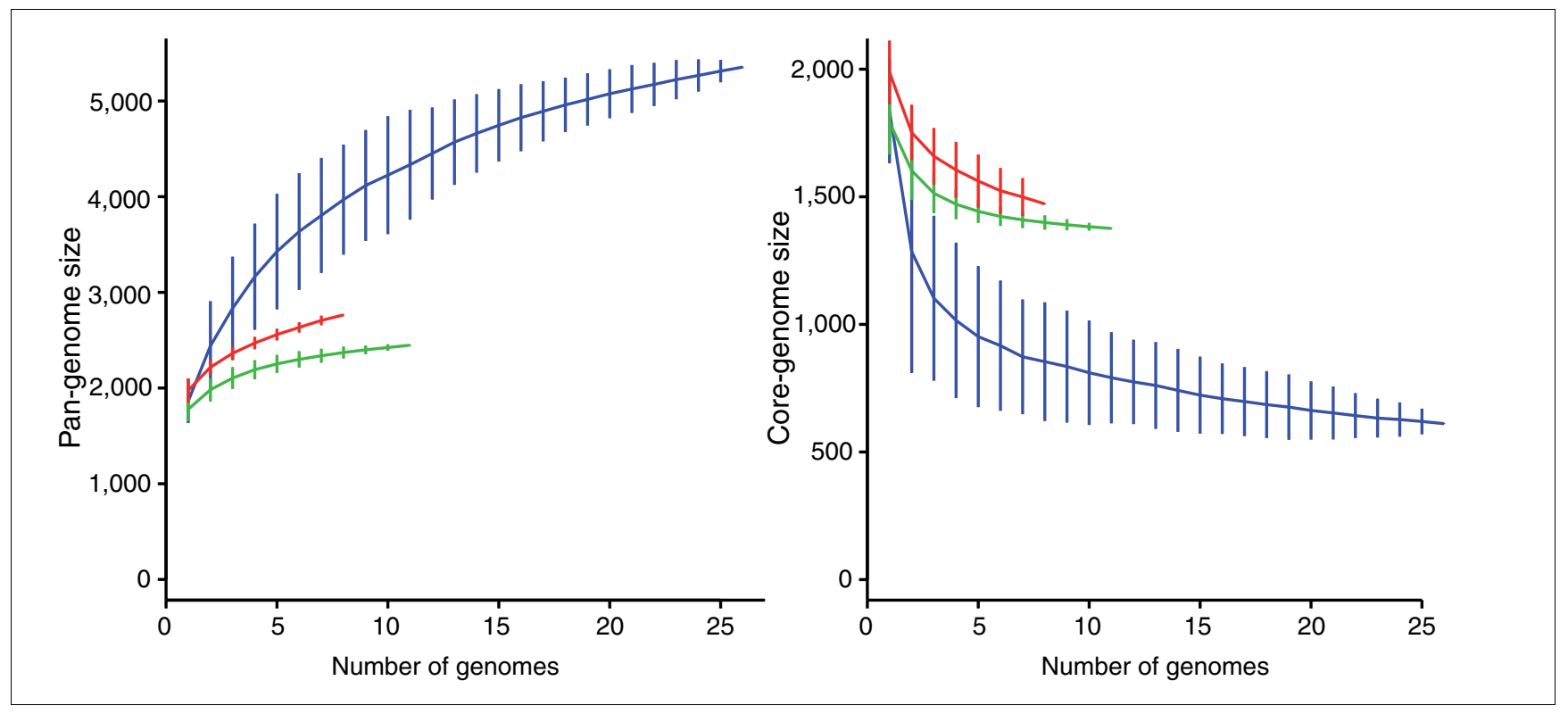

Figure 2

Accumulation curves for the total number of genes (left) or the number of genes in common (right) given a number of genomes analyzed for the different species of Streptococcus (in blue), the different strains of S. agalactiae (in red) and S. pyogenes (in green). The vertical bars correspond to standard deviations after repeating one hundred random input orders of the genomes. 


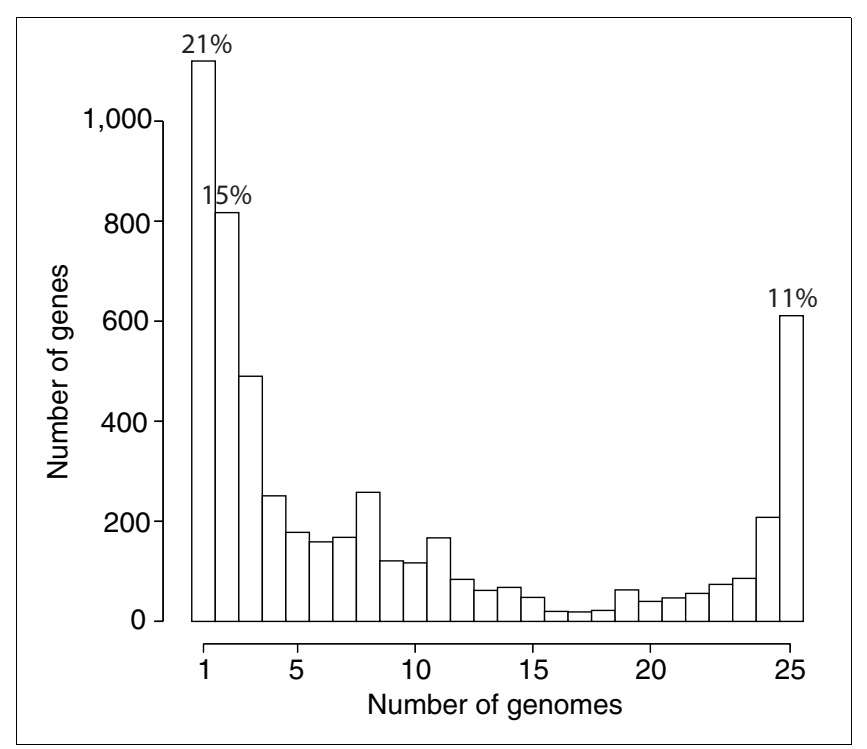

Figure 3

Frequency of genes within the 26 genomes included in this analysis. Genes present in a single genome represent lineage specific genes, while at the opposite end of the scale, genes found in all 26 genomes represent the Streptoccocus core-genome.

core-genome size does not level out, and appears as though it might still be influenced by the inclusion of new genome sequences. On the whole, these analyses suggest that it is possible to delineate a core-genome at both genus and species level. We analyzed four such core-genome data sets: the
Streptococcus core-genome (611 genes), and the coregenomes of $S$. agalactiae (1,472 genes), S. pyogenes (1,376 genes) and $S$. thermophilus (1,487 genes). To save computation time, the Streptococcus core-genome data set was reduced to ten taxa by keeping only two strains per species for S. agalactiae, S. pyogenes, S. thermophilus (strains A909 and NEM316, MGAS9429 and M1 GAS, and CNRZ1066 and LMG 18311, respectively). After discarding clusters of genes containing paralogs (that is, clusters containing more than one gene per taxon), and alignments with uncertain site homologies, we obtained four data sets containing 260, 1,297, 1,212 and 1,365 genes representing the alignable coregenomes of Streptococcus, S. pyogenes, S. agalactiae, and $S$. thermophilus, respectively.

Determinations of the number of genes gained and lost on each of the lineages shows considerable variation (Figure 4) and, in agreement with earlier studies, gene gain was generally considerably greater than gene loss, as well as being particularly evident on external branches [23]. The lineage in the interspecific analysis showing the greatest gene gain was $S$. suis, followed closely by $S$. pneumoniae and $S$. mutans. Even within a species, between strains, the numbers of genes gained and lost were very high, reaching, for example, values in excess of 150 for gene gain in S. agalactiae strain H36B. High levels of gene gain and loss were evident, even for closely related isolates of the same serotype in S. pyogenes (for example, M1 GAS/MGAS5005; SSI-1/MGAS315; MGAS9429/MGAS2096). Branch lengths of the $S$. pyogenes

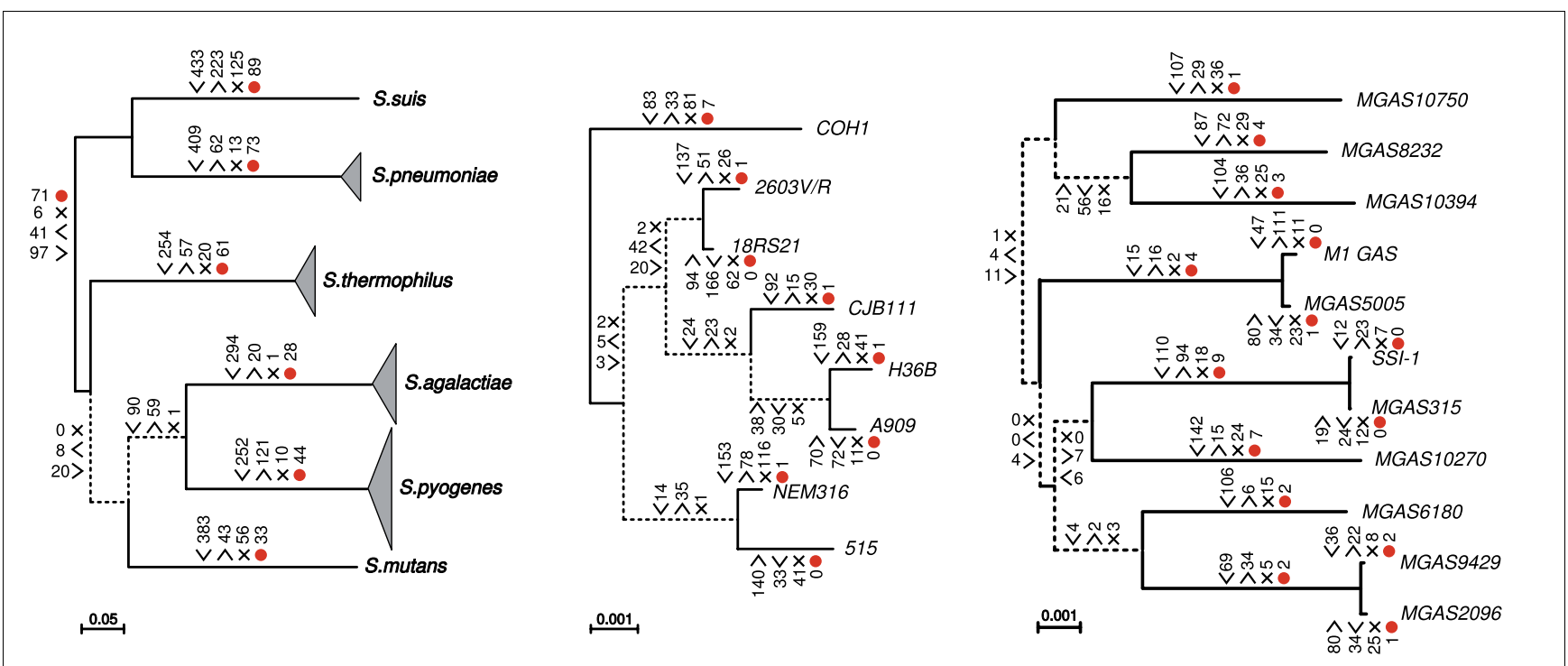

\section{Figure 4}

Gene gain, loss and duplication, and positive selection. Core-genome phylogenies of Streptococcus (left), S. agalactiae (middle), and S. pyogenes (right) based on concatenated genes. Dashed lines correspond to unresolved branches. Numbers adjacent to angle brackets facing the branch refer to genes gained, opposite direction - genes lost, and ' $x$ ' refers to duplicated loci. Values correspond to the most parsimonious unambiguous changes, following an equally penalized model (that is, gain, loss and duplication events cost the same numbers of changes). Numbers adjacent to the red dot correspond to the number of genes under positive selection within the core-genome, on a particular lineage. 
concatenated tree were much longer than those for $S$. agalactiae, suggesting the lineages might be much older; however, despite this there was generally more gene gain on the $S$. agalactiae branches than on $S$. pyogenes branches. Large values for duplications were also a feature of the lineage specific evolution (Figure 4). Phylogenetic analysis of several of these cases suggests this is a combination of lineage specific duplications as well as LGT events involving homologous sequences from other species of Streptococcus. When gene gain was penalized with respect to gene loss (for example, [24]), not surprisingly, it globally decreased the number of gene gains and increased the number of gene losses (Additional data file 3) and, as a consequence, increased the number of genes in the pan-genomes of ancestral nodes (data not shown). Nevertheless, even with a penalty, gene gain remained in excess of gene loss on some lineages (Additional data file 3 ).

\section{Recombination}

\section{Between species of Streptococcus}

The results of the approximately unbiased (AU) test indicated that 39 out of 260 genes rejected the concatenated tree. The $p$ value heatmap (Figure 5a) indicates that some gene trees showed the same or very similar histories, depicted by groups of topologies with a similar $p$ value pattern (for example, topologies 1 to 47, and 48 to 65). On the other hand, a small group of genes rejected most topologies (that is, genes 230 to 260, read horizontally in Figure $5 \mathrm{a}$ ), and at the same time, their trees were rejected by most of the genes (that is, topologies 230 to 260, read vertically in Figure 5a). Although different topologies were supported by various groups of genes, the majority of genes did not reject the concatenated tree and only a small subset of genes proposed significantly different trees. The analysis of bipartitions (Figure 5b) demonstrated that the vast majority of genes supported three distinct bipartitions, corresponding to the monophyly of $S$. pyogenes, $S$. pneumoniae and $S$. thermophilus (bipartitions 28, 29, and 30, respectively). Also generally supported were the monophyly of $S$. agalactiae, the monophyly of the group $S$. pneumoniae $+S$. suis, and the monophyly of the group $S$. agalactiae $+S$. pyogenes (bipartitions 27, 26 and 25, respectively). Several other bipartitions were only supported by some genes (for example, bipartition 19, corresponding to the grouping of $S$. pneumoniae with $S$. thermophilus), while others were only supported by one or a few genes (for example, bipartition 10 and 11). The well supported conflicting bipartitions figure (Figure $5 \mathrm{c}$ ) is a summary of the $p$ value heatmap (Figure $5 \mathrm{a}$ ) and bipartition analyses (Figure $5 \mathrm{~b}$ ). A majority of the genes (around 150 out of 260) show no conflict with each other. Most of them support the monophyly of the different species and the lineage $S$. pneumoniae $+S$. suis, and most of them do not reject the concatenated gene tree. Another set of genes showed some instances of conflict with the aforementioned set of 150 , but most of them were in conflict with each other. They tend to support the same principal groups as the set of 150 , with a few additional bipartitions that are conflict- ing. A final group of genes conflict with the first and the second group, as well as with each other, corresponding to genes that rejected most of the other gene trees in the AU test (Figure 5a) and that provide support for rare bipartitions; genes of this set have strongly incongruent histories with the other genes (for a detailed list, see Additional data file 4). The topologies used to test for positive selection were the concatenated gene tree for the genes that don't reject it, and individual gene trees for those loci that do reject the concatenated tree.

\section{Within S. agalactiae}

The concatenated gene tree was rejected by 750 genes of the core-genome of $S$. agalactiae. On the whole, most genes rejected most of the other gene trees (Figure 6a), although there were also some genes that did not reject the majority of gene trees. There were no commonly well supported bipartitions across the genes (Figure 6b). Around half of the genes provided either no, or only weak, bootstrap support for any bipartition (genes 1 to 560 ; Figure 6b), while the rest of the genes supported different sets of bipartitions. The most commonly supported groups of strains were $515+\mathrm{NEM} 316$, $\mathrm{A} 909+\mathrm{H} 36 \mathrm{~B}, \quad 515+\mathrm{NEM} 316+\mathrm{COH} 1, \mathrm{~A} 909+\mathrm{CJB} 111+\mathrm{H} 36 \mathrm{~B}$, A909+CJB111+H36B, and 515+COH1 (bipartitions 75 to 70 , respectively; Figure 6b). Additional, numerous bipartitions were supported by only one or a few genes. Because they possessed a too limited phylogenetic signal, around half of the genes (genes 1 to 560) showed no conflict with any of the other genes (Figure 6c). Although the AU test suggested that some of these genes have different histories, it is difficult to reach any definitive conclusions about the congruence of these gene histories since phylogenetic signal was so limited or absent (genes with no sequence divergence between strains).

The second half of the core-genome can be split into two groups. The first group contains genes that have some conflict with each other, and that tend to support the six bipartitions described earlier, plus three additional ones. The second group contained genes that were largely in conflict with each other, and with the preceding group. This latter group provided support for a number of rarely supported bipartitions. While the first group contained genes that had only partly incongruent histories (only a few bipartitions in conflict), genes of the last group had more incongruent gene histories (greater number of bipartitions in conflict). Given these results, and the ambiguity of defining which genes had the same history, we analyzed each gene with its own gene tree in the subsequent positive selection analyses.

\section{Within S. pyogenes}

As for S. agalactiae, while a few genes rejected nothing, the majority of genes rejected the other gene trees (Figure 7a). Three bipartitions were generally supported, although not always, and with various bootstrap scores, corresponding with serotype groupings: MGAS5005+M1 GAS, MGAS315+SSI-1, and MGAS2096+MGAS9429 (bipartitions 


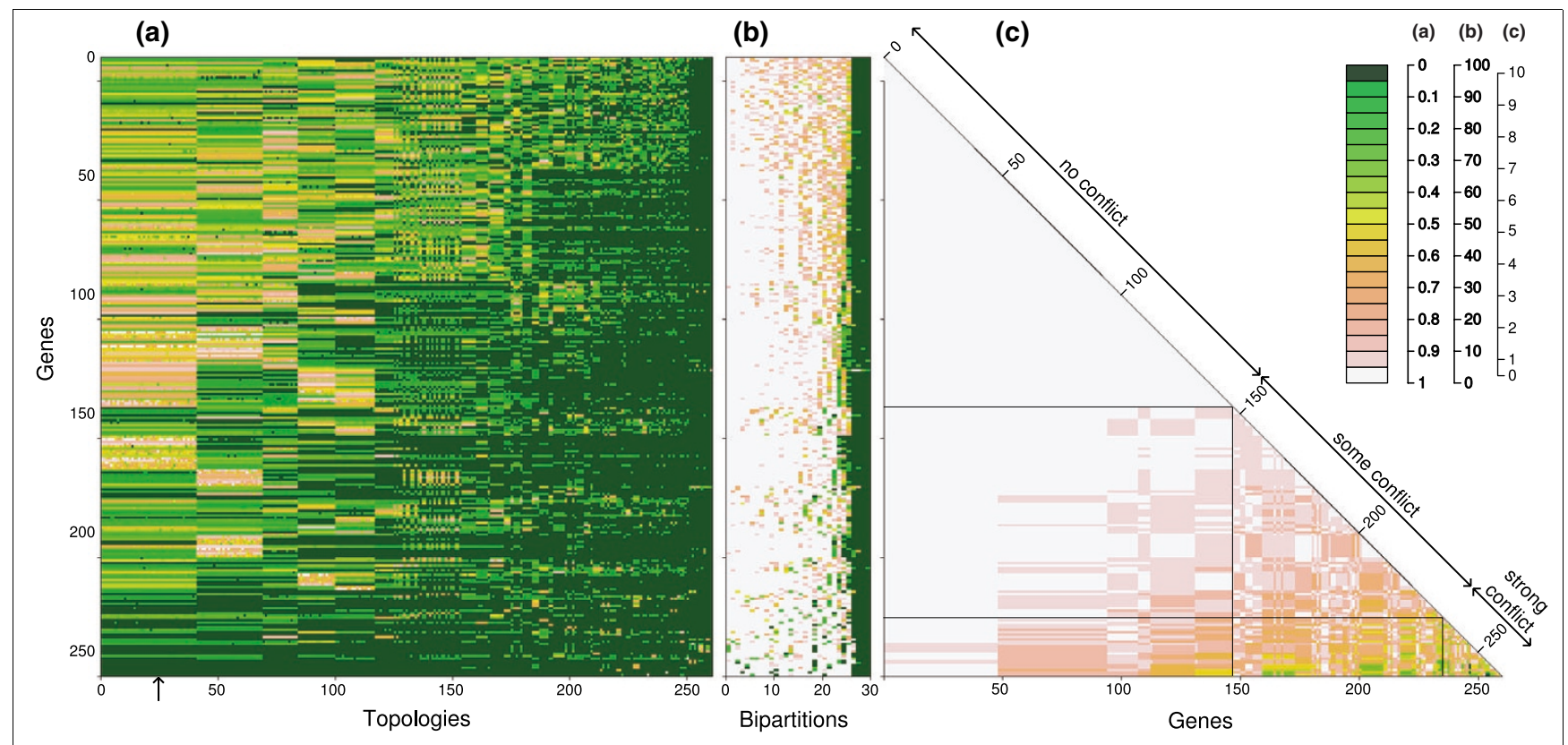

\section{Figure 5}

Streptococcus recombination heatmaps. Heatmaps of the (a) AU test, (b) bipartitions bootstrap scores and (c) well supported conflicting bipartitions on the core-genome of Streptococcus. Topologies are ordered from the less rejected (on the left) to the most rejected (on the right). Bipartitions are ordered from the less supported (on the left) to the most supported (on the right), and only bipartitions supported by at least a 70\% bootstrap score are represented. Genes are ordered from the less conflicting (left and top) to the most conflicting (right and bottom). The well supported conflicting bipartitions heatmap represents a symmetrical distances matrix, where each cell corresponds to the number of well supported (that is, bootstrap $\geq 90$ ) conflicting bipartitions between two genes. A color key is given on the right side, and gradations correspond to $p$ values, bootstrap percentages, and number of conflicting bipartitions, left to the right respectively. The arrow locates the concatenated tree.

131 to 129 , respectively; Figure $7 \mathrm{~b})$. A total of 434 genes tended to also provide support for various unique bipartitions. Around half of the genes had weak or no phylogenetic signal, and, as a consequence, had no conflict with any other trees (Figure 7b). A set of around 200 genes, most of which

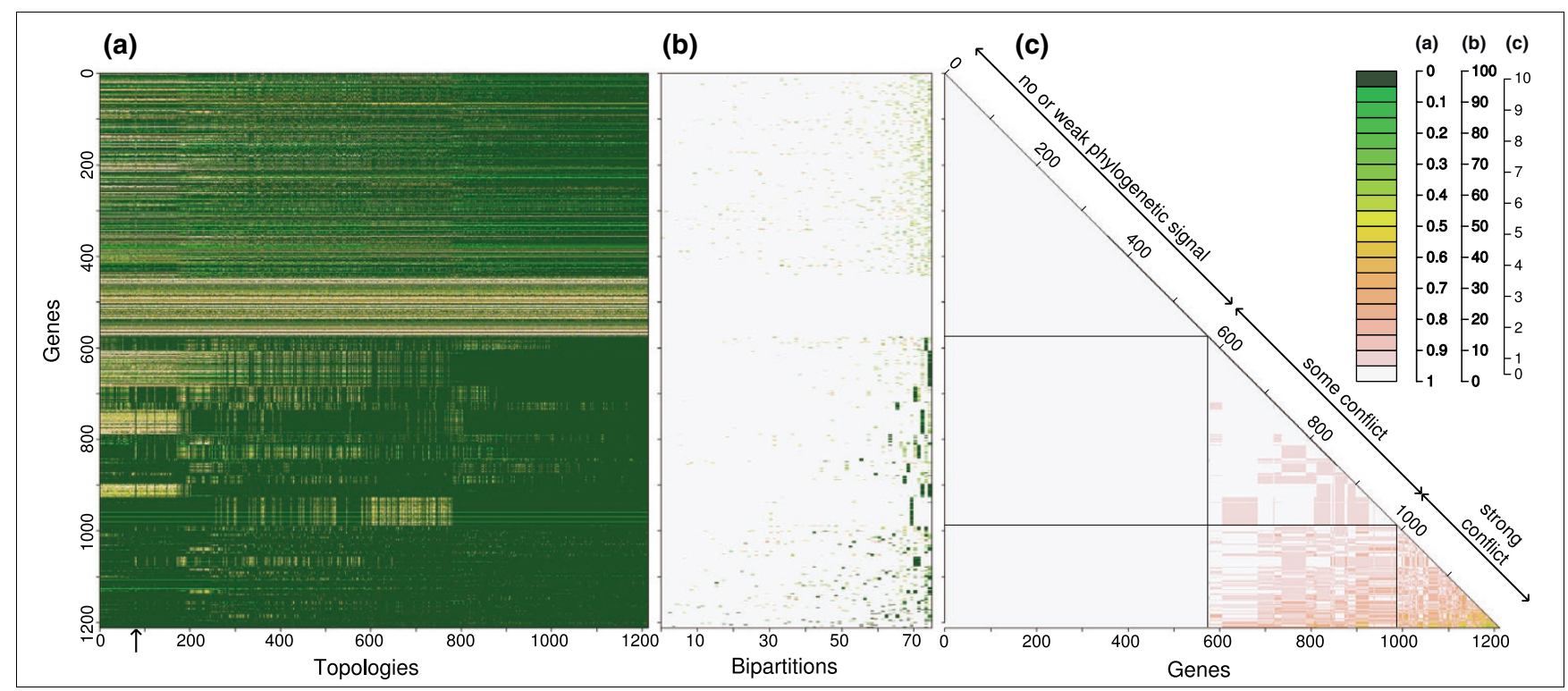

Figure 6

S. agalactiae recombination heatmaps. The layout is the same as Figure 5 but for the core-genome of S. agalactiae. 


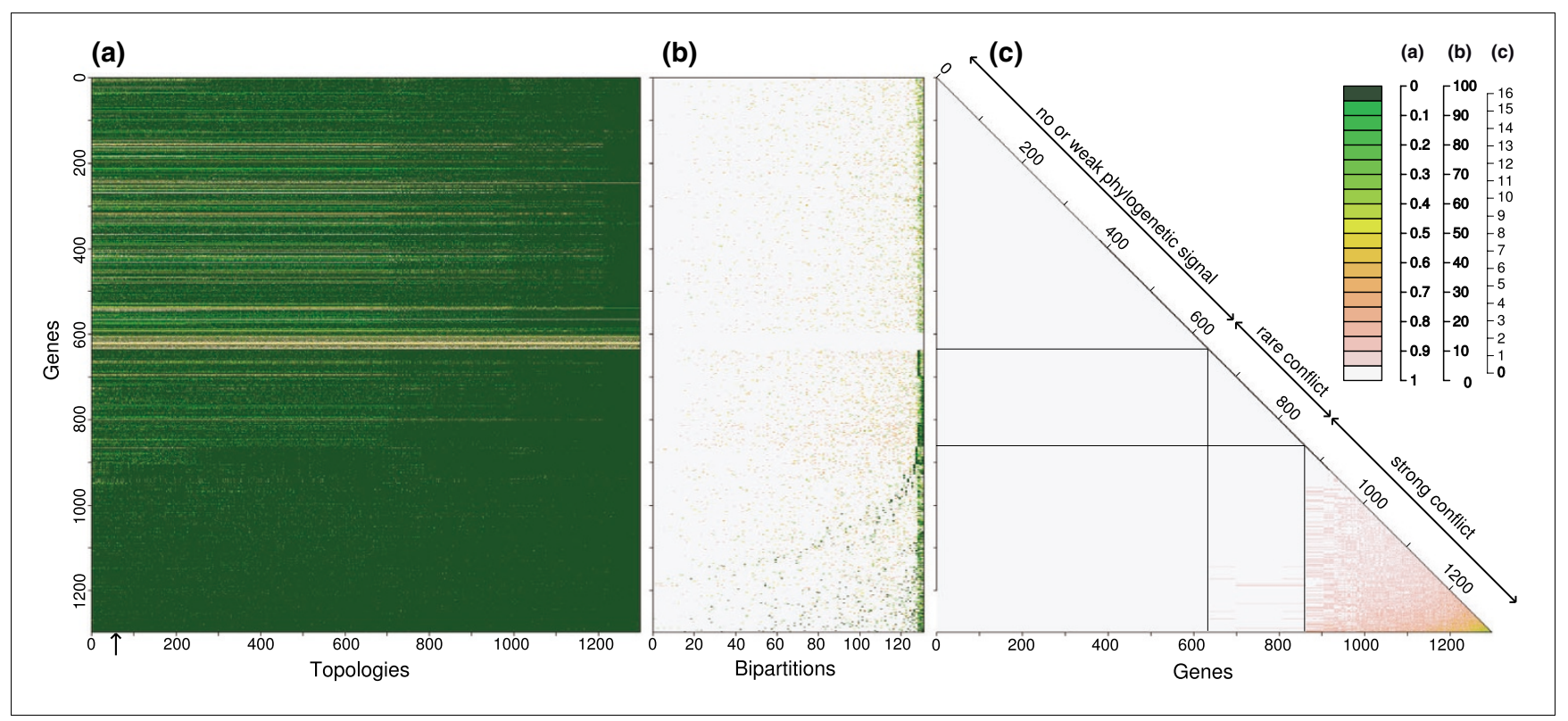

Figure 7

S. pyogenes recombination heatmaps. The layout is the same as Figure 5 but for the core-genome of $S$. pyogenes.

supported the three bipartitions detailed above, tended not to conflict with each other, but occasionally with the final grouping of genes. This latter group was composed of the 434 genes mentioned above, which supported variously different bipartitions, and thus tended to be in conflict with each other. Overall, the S. pyogenes core-genome is composed of genes that are largely congruent for a portion of relatively recent history (that is, the serotype monophyly), while one-third of the core-genome appears to have strongly incongruent histories for older events. Because it appeared difficult to define which genes were likely to have the same history, we analyzed each gene with its own gene tree in the subsequent positive selection analyses.

\section{Substitution analysis of recombination}

The pairwise homoplasy index (PHI) approach suggested that around $20 \%$ of the genes were recombinant within the coregenome of Streptococcus and $S$. pyogenes, while within $S$. agalactiae only about $3 \%$ of the genes were recombinant (Table 2). Employing a more conservative approach that considers as recombinant only those genes found by three differ- ent substitution approaches (PHI, MaxChi and neighbor similarity score (NSS)), these proportions were reduced, but the relative differences between the data sets remained (Table 2). With the phylogenetic approach detailed above, numerous genes had weak phylogenetic signal, and several groups of genes were only partially incongruent; therefore, it can be difficult to define clearly which genes have different histories. It is, however, possible to adopt a conservative approach that considers as putative recombinants only those genes with strong phylogenetic incongruence (SPI), with most of the other genes. Nevertheless, only a small proportion of genes was identified by both PHI and SPI approaches as putative recombinants (Table 2), suggesting that each approach tends to identify different types of recombination event. We therefore propose that an estimate of the complete set of putative recombinants can best be considered as the set of genes identified by SPI plus the genes identified by all three substitution recombination methods (Table 2). This yields an estimate of $18 \%$ of the core-genome for $S$. agalactiae as putative recombinants, $19 \%$ for the genus Streptococcus, and $37 \%$ for S. pyogenes.

Table 2

Number of genes showing evidence of recombination

\begin{tabular}{lccccc}
\hline & I. SPI & 2. PHI & 3. PHI $\cap$ MaxChi & I $\cap 2$ & I $\cap 3$ \\
\hline Between species & $26(10.0 \%)$ & $54(20.8 \%)$ & $35(13.5 \%)$ & $11(4.2 \%)$ & $53(19.2 \%)$ \\
S. pyogenes & $434(33.5 \%)$ & $284(21.9 \%)$ & $168(12.9 \%)$ & $186(14.3 \%)$ & $477(36.8 \%)$ \\
S. agalactiae & $222(18.3 \%)$ & $34(2.8 \%)$ & $7(0.6 \%)$ & $18(1.5 \%)$ & $223(18.4 \%)$ \\
\hline
\end{tabular}


Table 3

\begin{tabular}{|c|c|c|c|c|}
\hline \multicolumn{5}{|c|}{ Genes under positive selection } \\
\hline Data set & Lineage & $\mathrm{n}$ & PS & $\%$ \\
\hline \multirow[t]{7}{*}{ Streptococcus } & S. mutans & 260 & 33 & 12.69 \\
\hline & S. pneumoniae & 260 & 73 & 28.08 \\
\hline & S. suis & 260 & 89 & 34.23 \\
\hline & S. thermophilus & 260 & 61 & 23.46 \\
\hline & S. agalactiae & 260 & 28 & 10.77 \\
\hline & S. pyogenes & 260 & 44 & 16.92 \\
\hline & (S. pneumoniae, S. suis) & 221 & 71 & 32.13 \\
\hline \multirow[t]{8}{*}{ S. agalactiae } & $\mathrm{COHI}$ & 1,212 & 7 & 0.58 \\
\hline & I8RS2I & 1,212 & 0 & 0.00 \\
\hline & NEM3 16 & 1,212 & 1 & 0.08 \\
\hline & $\mathrm{H} 36 \mathrm{~B}$ & 1,212 & 1 & 0.08 \\
\hline & A909 & 1,212 & 0 & 0.00 \\
\hline & $2603 V / R$ & 1,212 & 1 & 0.08 \\
\hline & CJBIII & $1,2 \mid 2$ & 1 & 0.08 \\
\hline & 515 & 1,212 & 0 & 0.00 \\
\hline \multirow[t]{14}{*}{ S. pyogenes } & MGASI0270 & $\mathrm{I}, 297$ & 7 & 0.54 \\
\hline & MGASI0394 & $\mathrm{I}, 297$ & 3 & 0.23 \\
\hline & MGASI0750 & $\mathrm{I}, 297$ & 1 & 0.08 \\
\hline & MGAS2096 & I,297 & 1 & 0.08 \\
\hline & MGAS3I5 & $\mathrm{I}, 297$ & 0 & 0.00 \\
\hline & MGAS5005 & $\mathrm{I}, 297$ & 1 & 0.08 \\
\hline & MGAS6I80 & $\mathrm{I}, 297$ & 2 & 0.15 \\
\hline & MGAS8232 & $\mathrm{I}, 297$ & 4 & 0.31 \\
\hline & MGAS9429 & $\mathrm{I}, 297$ & 2 & 0.15 \\
\hline & MI GAS & $\mathrm{I}, 297$ & 0 & 0.00 \\
\hline & SSI-I & 1,297 & 0 & 0.00 \\
\hline & (MGAS9429, MGAS2096) & 925 & 2 & 0.22 \\
\hline & (MGAS5005, MI GAS) & 978 & 4 & 0.41 \\
\hline & (SSI-I, MGAS3I5) & 983 & 9 & 0.92 \\
\hline \multirow[t]{3}{*}{ S. thermophilus } & CNRZI066 & 1,365 & 3 & 0.22 \\
\hline & LGM I83II & 1,365 & 3 & 0.22 \\
\hline & LMD-9 & 1,365 & 14 & 1.03 \\
\hline
\end{tabular}

PS, positive selection.

\section{Positive selection analysis}

The number of genes that showed evidence for positive selection was particularly high within the Streptococcus coregenome (between $10 \%$ and $40 \%$; Table 3). The $S$. pneumoniae and $S$. suis lineages, and the ancestral lineage leading to these two species, exhibited the greatest proportion of the coregenome evolving under positive selection (28\%, 34\% and $32 \%$, respectively; Table 3). Approximately one-third of the genes showed positive selection on only one lineage, and no gene was selected in all possible lineages (Figure 8). There were, however, many examples of genes selected on multiple lineages, including several genes selected on as many as 5 (12 genes) or 6 (4 genes) different lineages (Figures 8 and 9; see Additional data file 5 for a complete list of all genes and lineages under positive selection). A significant proportion of positively selected genes for $S$. suis, $S$. pneumoniae, and $S$. thermophilus was uniquely selected on each of these lineages (21\%, 19\%, and 24\%, respectively), in contrast to that for $S$. agalactiae, S. pyogenes, and S. mutans, which had either no uniquely selected loci ( $S$. agalactiae), or a very small proportion (Figure 9). Analysis of variance of genes under positive selection pressure supported a significant effect of both lineage and biochemical main role category (Table 4). Post hoc multiple comparisons showed that the main effect was due to two categories, 'DNA metabolism' and 'Transcription'. Less strongly supported, but still significant, was the interaction between lineages and main role categories (Table 4). This interaction appeared mainly due to an increase of genes under positive selection for loci involved in transcription, protein fate, protein synthesis and DNA metabolism for 
Table 4

\begin{tabular}{|c|c|c|c|c|c|}
\hline & Df & Sum Sq & Mean Sq & $F$ value & $p$ value \\
\hline Lineage & 6 & 2,954 & 492 & 23.9 & $<0.0001$ \\
\hline Main role & 10 & 1,086 & 109 & 5.27 & $<0.0001$ \\
\hline Interaction & 60 & 1,974 & 33 & 1.6 & 0.003 \\
\hline Residuals & 1,699 & 35,005 & 21 & & \\
\hline
\end{tabular}

Df, degree of freedom.

the $S$. pneumoniae-S. suis ancestral lineage and the $S$. suis lineage.

In addition to identifying genes and lineages under positive selection, the branch-site test also identifies sites using a Bayes empirical Bayes approach [25]. For $91 \%$ of the genes under positive selection, specific sites were proposed (posterior probability >0.95). Interestingly, when a gene was independently selected on different lineages, the sites under positive selection were generally not the same across lineages, arguing for different selection pressure located at different sites. In contrast to the interspecific comparisons, positive selection was evident for only a few genes within the coregenome, across strains of the different Streptococcus species (Table 3, Additional data file 5), including a few lineages that showed slightly increased levels of positive selection relative to the rest. For S. agalactiae the exceptional lineage was $\mathrm{COH} 1$, for $\mathrm{S}$. pyogenes the exceptional lineages were MGAS10270 and that leading to SSI-1/MGAS315, and for S.

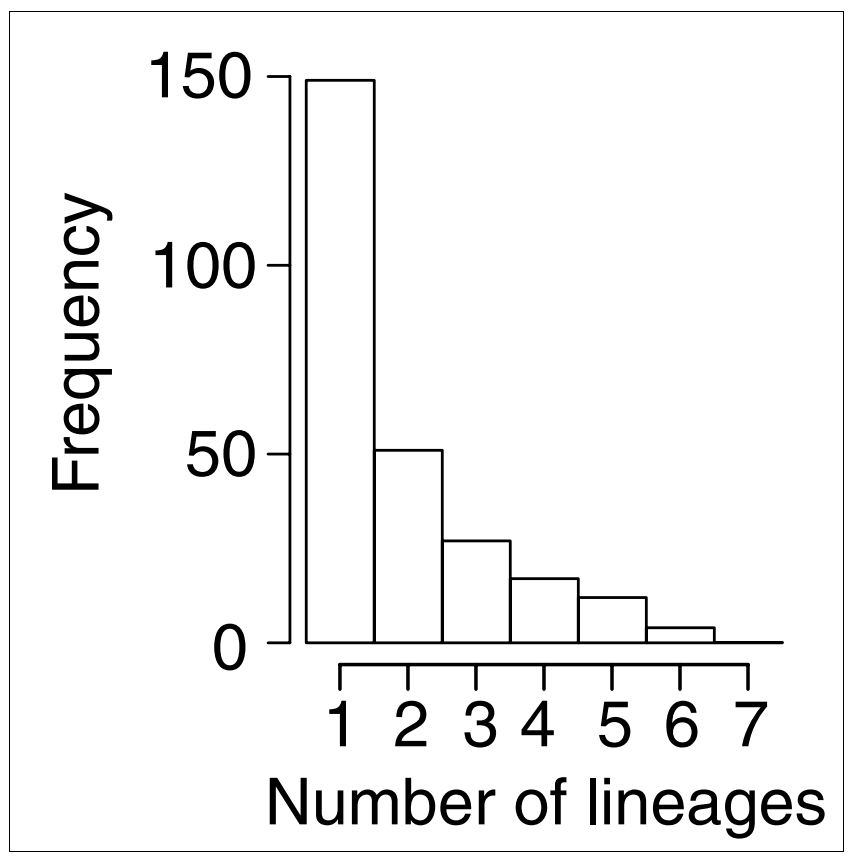

Figure 8

Frequency of positive selection. Numbers of genes showing evidence of positive selection in $1-7$ lineages. thermophilus it was LMD-9. A significant number of genes evolving under positive selection were also judged as putative recombinants (Table 5). This was particularly true for the $\mathrm{S}$. pyogenes genome, where $78 \%$ of the genes under positive selection were putative recombinants. Approximately half of these genes were identified as recombinants by the substitution based recombination methods, and the other half by the phylogenetic approach.

\section{Discussion}

Core-genome, pan-genome, and recombination

We estimate that the pan-genome of the genus Streptococcus probably exceeds at least three times the average genome size of a typical Streptococcus species. This huge variability in gene content between species is also evident in comparisons across strains of the same species. Our prediction for the $S$. agalactiae pan-genome is in general agreement with that of Tettelin et al. [22]. The marked difference in estimated pangenome size for these two species may be a reflection of their habitat differences. The human oral-nasal mucosa is the primary habitat for $S$. pyogenes, whereas $S$. agalactiae was first identified as a bacteria linked to bovine mastitis, and later in humans, where it colonizes the lower gastrointestinal tract and vaginal epithelium of healthy adults. This apparent broader habitat range for $S$. agalactiae, and presumably, therefore, a greater available gene pool for lateral gene transfer, could explain the difference in pan-genome size of these two species.

The pronounced evolutionary flexibility of these bacterial genomes is further evident in the determinations of gene gain, loss and duplication on each of the respective lineages. Gene gain figures were generally higher for $S$. agalactiae than for $S$. pyogenes, despite the fact that branch lengths suggest the $S$. pyogenes lineages may be older, and is likely a consequence of the overall smaller pan-genome size for $S$. pyogenes. For some species, gene gain figures exceeded $20 \%$ of the total gene content for the organism. Our results in this regard are in general agreement with those of Hao and Golding [23], while also extending the estimates to additional taxa of Streptococcus, and lineages of S. agalactiae and S. pyogenes, and we would certainly concur with these authors that much of this gene gain likely reflects species specific adaptation. In our opinion, a plausible explanation of the discrep- 


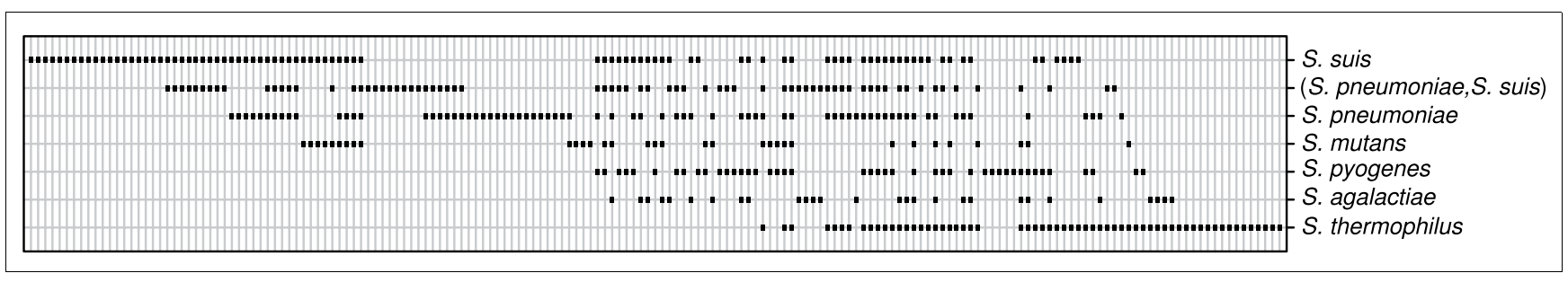

Figure 9

Positive selection occurrence per genes and lineages. A black dot indicates positive selection. The genes and lineages were ordered following a correspondence analysis.

ancy between gene gain and loss is that much of the pangenome remains unsampled, and, therefore, we simply cannot detect many gene loss events, resulting in an underestimate of that category. In several genome reconstruction analyses, gene gain is penalized with respect to gene loss (for example, [24,26-28]). This procedure logically results in an increase of gene loss relative to gene gain. It has the direct consequence of increasing ancestral genome sizes, and reducing the importance of LGT events to the benefit of genome reduction processes. Nevertheless, this approach is questionable when one attempts to reconstruct genome composition, and in particular, for the case of Streptococcus. First, because the genome size is relatively stable in Streptococcus, the ancestral genome sizes were arguably of the same order. Second, the number of genome specific genes is so common that there is little reason to postulate that gene gain is less probable than gene loss. Therefore, at least in the case of Streptococcus, we question the validity of an approach that favors genome reduction processes over LGT. In that regard, the development of probabilistic models will be highly valuable to estimate rates of gene gain and loss (for example, $[16,29]$ ).

In contrast to the pan-genome, the core-genome size of the taxonomic groups included in our analysis are much better estimated. Within Streptococcus, we characterized several core-genomes, the composition of which depended on the taxonomic level considered. Our prediction of core-genome composition for $S$. agalactiae was in general agreement with Tettelin et al. [22] and Brochet et al. [30]. The slight differences in the absolute numbers between the three studies are due to differences in methodology used to define orthology [22], or the use of DNA microarray hybridization data [30]. At the genus level, the core-genome corresponded to $25 \%$ of a typical Streptococcus genome, while at the species level it represented around $60 \%$ of the genome. Earlier studies involving other groups of bacteria have suggested that such core-genomes may be relatively free of recombination [3133]. If you consider the union of both substitution based methods and phylogenetic based methods we estimate that around $18 \%$ of the core genome of the genus Streptococcus is recombinant and as much as $35 \%$ of the genome of $S$. pyogenes. In addition to the fact that we are analyzing a different group of taxa, and thus levels of recombination might well be expected to be different, our results differ from these earlier estimates, also because of approach. We concur with the comments of Susko et al. [34] that attempts to evaluate phylogenetic congruence of core-genes need to involve comparisons with as many relevant topologies as possible, and not just that favored by the concatenated topology, some variants of it, or particular canonical markers such as the small subunit rRNA. In doing this, however, we think it is important to keep in mind that topologies may be rejected as being congruent, even though the genes may provide little phylogenetic signal, and, thus, only that proportion of the gene trees that is rejected based on strongly supported conflicting nodes should be regarded as incongruent. This was particularly evident in our analysis of $S$. pyogenes, where the vast majority of topologies reject one another despite the fact that at least half of these genes have little or no phylogenetic signal. Furthermore, our assessment of core genome recombination also differs from some of these earlier studies by the inclusion of substitution based approaches to recombination detection. There was little overlap in the loci identified as putative recombinants using both the phylogenetic and the substitu-

Table 5

Total number of genes showing evidence of positive selection and recombination

\begin{tabular}{lcccc}
\hline Data set & PS & PS and R & PS and SPI & PS and IR \\
\hline Between species & 175 & $43(25 \%)$ & $20(8 \%)$ & $29(11 \%)$ \\
S.agalactiae & 10 & $4(40 \%)$ & $4(40 \%)$ & $0(0 \%)$ \\
S.pyogenes & 32 & $25(78 \%)$ & $21(65 \%)$ & $17(53 \%)$ \\
\hline
\end{tabular}

PS, positive selection; $R$, recombination. 
tion based approaches, suggesting they were identifying very different types of recombination. The substitution based approaches are likely to detect homologous recombination of smaller pieces of DNA that could be missed by a phylogenetic approach. The more restricted habitat distribution for $S$. pyogenes may also be an explanation for the elevated amounts of recombination in the core-genome of that species. A more reduced gene pool for possible recombination would not only result in a smaller pan-genome size (as suggested above for $S$. pyogenes), but it would also result in the propensity for more homologous recombination of core-genome components, at least partly because the relative proportion of conspecific donor pieces of DNA are likely to be greater.

\section{Positive selection in the core-genome}

A logical surmise often made in studying pathogen evolution is that much of the host specific adaptation that a bacterial species exhibits will be associated with its species specific genes. Perhaps as a consequence of this common sense viewpoint, adaptation within the core genome has received much less attention. Our analysis reveals that during the diversification of the genus Streptococcus there has been significant amounts of positive selection pressure on core genome components and that this selection pressure has occurred disproportionately in certain lineages, and biochemical categories. Such an important positive selection signature within the core-genome of Streptococcus is perhaps somewhat surprising, as these predominately housekeeping genes might be expected to evolve under strong purifying selection. At the same time, we would argue that many of these genes are undoubtedly related to the colonization, persistence, survival, and propensity to cause disease in these organisms and thus are associated with the adaptive specifics of the bacteria.

Based on currently available phylogenies for the genus Streptococcus [35], there appears to be a rough correlation with the relative divergence of taxa and the level of positive selection detected in different lineages. For example, $S$. pyogenes and $S$. agalactiae are both members of the Pyogenes taxonomic group and there are fewer numbers of genes selected on these lineages than for taxa from different taxonomic groups. Several genomes also tend to resemble one another in relation to the genes that were positively selected, while others, such as $S$. suis and $S$. thermophilus, exhibit higher levels of specific adaptation. $S$. suis was also the species with the largest number of positively selected genes in its core-genome, relative to the other lineages, and the genome that had the greatest amount of gene gain and loss incurred since the separation of the $S$. pneumoniae-S. suis common ancestor. This suggests a lineage that has been under strong selection pressure, both with regard to acquiring new genes and with regard to the sequence characteristics of the core genome components. This selection pressure is undoubtedly correlated with particular characteristics of the host species, swine. Current phylogenies support $S$. acidominimus as the sister group to $S$. suis [35], a species associated with bovine vagina, the skin of calves, and raw milk, suggesting the possibility of host divergence in one or another (or possibly both) lineage, subsequent to their split from a common ancestor. $S$. suis is also known as an occasional zoonotic pathogen of humans, causing septicemia, meningitis, endocarditis [36], and, most recently, streptococcal toxic shock syndrome [15]. Our analysis suggests an apparent evolutionary flexibility of the $S$. suis genome that could perhaps be related to this propensity for host jumping.

In addition to the lineage effect in the interspecific selection analysis, there was also an effect of biochemical roles, with DNA metabolism and transcription significantly different from the majority of the other categories and correlated with higher incidence of positive selection. An excess of positive selection in the genes related to transcription is perhaps surprising, as these genes are generally well conserved and are known to be particularly recalcitrant to recombination. Furthermore, the $S$. suis and S. pneumoniae-S. suis ancestral lineages showed a disproportionate amount of positively selected genes for several biochemical categories. Thus, the extent of adaptive molecular evolution varies across lineages and gene roles, undoubtedly reflecting the habitat heterogeneity of the genus Streptococcus. Our results also reveal that positive selection was partly linked to recombination. Recombination can create artifactual positive selection results [37], particularly for genes showing evidence of intragenic recombination. This is less probable for genes identified as recombinant using the phylogenetic recombination detection procedure, because these loci were analyzed for positive selection with their own phylogenies, thereby taking into account possible LGT events. It is possible that many LGT loci could also be positively selected because of strong selective forces that might be expected to act on newly acquired genes. Marri et al. [16] found that many species specific genes within the Streptococcus genus showed evidence of adaptive evolution, and concluded that LGT played an important adaptive role.

Although a good deal of positive selection pressure was evident in the analysis of different lineages within the genus Streptococcus, there was much less evidence for positive selection between the different lineages of $S$. agalactiae, $S$. pyogenes, and $S$. thermophilus. Nevertheless, these absolute numbers of genes under positive selection are not corrected for depth of ancestry. In other words, they do not reflect rates of adaptative mutations for specific lineages; instead, they represent the core-genome fraction that has participated in the adaptation of a specific taxon. There were, however, several lineages in each of these species that had slightly elevated levels of selection relative to the rest. In $S$. pyogenes, for example, the lineage leading to the M3 serotype had nine genes under positive selection, while the majority of other lineages had two or less (see Additional data file 5 for a complete description of these genes). Compared to other M types, sero- 
type M3 strains cause more cases of invasive disease, such as necrotizing fasciitis, bacteremia, and streptococcal toxic shock syndrome [38-42], a higher rate of lethal infections [4143], and exhibit occasional epidemic tendency [38]. The nine genes we identified as positively selected included loci implicated as virulence determinants in other species of Streptococcus, such as adenylosuccinate lyase, a homotetramer that catalyzes two discrete reactions in the de novo synthesis of purines, and has recently been implicated as a virulence factor for infective endocarditis, a serious endovascular infection caused by Streptococcus sanguinis [44], as well as genes involved in cell envelope, such as $U D P-N$-acetylmuramoylalanyl-D-glutamyl-2,6-diaminopimelate-D-alanyl-D-alanyl ligase, demonstrated to be integral to peptidoglycan biosynthesis and cell growth [45]. In addition to the evaluation of genes unique to M3 strains (for example, [46]), we suggest that these nine core-genome loci uniquely under positive selection pressure in this M3 lineage should be considered as putatively important in the unique pathogenic features of M3 strains.

In the case of $S$. agalactiae, the lineage that stood out from the rest with regard to levels of positive selection pressure was $\mathrm{COH} 1$, which is serotype III, ST17, significantly associated with neonatal invasive disease [47], and is hypothesized to have recently arisen from a bovine ancestor [48]. The seven genes selected on this lineage include a number of loci either already implicated in virulence in other bacteria, or for which there is some reason to suspect them as candidate virulence loci (see Additional data file 5 for a full description of these genes). For example, adenylosuccinate lyase, discussed above with regard to $S$. pyogenes, was also positively selected in this lineage. Phosphate acetyltransferase was uniquely selected along this $\mathrm{COH} 1$ lineage, and has recently been implicated in virulence in Salmonella enterica [49]. Also selected were a protein involved in the cell envelope, as well as two different $\mathrm{ABC}$ transporters. The cell envelope is a key overall component of virulence in $S$. agalactiae [50]. ABC transporters have been known for some time to be efflux mechanisms of drug resistance, although such efflux pumps are now also known to have physiological roles, conferring resistance to natural substances produced by the host, as well as possible roles in pathogenicity [51]. Perhaps the proposed recent shift in host preference from bovine to human for this lineage [48] is facilitated by molecular adaptation of $\mathrm{ABC}$ transporters that confer resistance to natural substances of the new host.

\section{Conclusion}

The research presented here employs a comparative genomics approach to define the core-genome component of the genus Streptococcus, as well as that of S. agalactiae, S. pyogenes, and $S$. thermophilus. We then assess levels of recombination and positive selection pressure in this core-genome for each of these taxonomic groups. Concomitant with these assessments of core-genome were estimates of the pangenome size of each of these groups, and levels of gene gain, loss and duplication on each of the lineages.

The pan-genome size of $S$. pyogenes appears to be quite well estimated with the 11 sequences currently available, and is approximately 2,500 genes. The pan-genome size of $S$. agalactiae is less well estimated with available sequence data and is in excess of 2,800 genes. Similarly, the pan-genome size of the genus Streptococcus is not accurately estimated with the 26 genomes analyzed here, and is in excess of 5,300 genes. We suggest that the broader habitat range for $S$. agalactiae may provide a greater available gene pool for lateral gene transfer, and could explain the difference in pan-genome size of $S$. agalactiae and $S$. pyogenes.

The core-genome components of each of these taxonomic groups is much better represented, and contrary to some earlier studies involving other groups of bacteria, which have suggested that such core-genomes may be relatively free of recombination, we estimate that around $18 \%$ of the coregenome of the genus Streptococcus is recombinant and as much as $35 \%$ of the genome of $S$. pyogenes. An explanation for the greater amount of recombination in $S$. pyogenes may be related to the more restricted habitat distribution for $S$. pyogenes, which would result in the propensity for more homologous recombination of core-genome components because the relative proportion of conspecific donor pieces of DNA is likely to be greater. Positive selection across the coregenome was particularly evident in the analysis of the different species within the genus Streptococcus, and it occurred disproportionately in certain lineages, as well as biochemical categories. $S$. suis was the lineage that showed the greatest positive selection pressure, the largest number of loci uniquely selected, and the lineage that had the greatest amount of gene gain and loss. In addition to the lineage effect in the interspecific selection analysis, there was also an effect of biochemical role, with genes related to DNA metabolism and transcription showing a significantly higher number of genes under positive selection. Contrary to the interspecific analysis, the selection analysis on individual species supported much less evidence for positive selection, but suggested there were particular lineages in each species that had experienced more core-genome selection pressure than the others. In the case of $S$. pyogenes this was the lineage leading to the M3 serotype, and we suggest that the nine genes identified as positively selected should be considered as putatively important in the unique pathogenic features of M3 strains. In the case of $S$. agalactiae the lineage with the disproportionate selection pressure was $\mathrm{COH} 1$, which is known to be significantly associated with neonatal invasive disease, and is hypothesized to have recently arisen from a bovine ancestor. We suggest that this proposed recent host jump from bovine to human for this lineage could be the explanation for the greater amount of selection pressure observed in this genome. 
Overall, this study indicates that there has been considerable recombination and positive selection pressure in the diversification of the Streptococcus core-genome, particularly at the interspecific level. Positive selection seems to be of principal importance in species differentiation and adaptation to new hosts, while it plays a less important role during strain evolution, where the process may be too slow to facilitate rapid strain adaptation. On the other hand, the process of recombination, through either LGT or homologous intragenic recombination, involving both the core-genome and the pangenome, appeared to be of main importance at a variety of evolutionary time scales. It seems likely that recombination is a more efficient means of change, ultimately making it the more universal process of Streptococcus adaptation. Although the cause-effect explanations are not necessarily clear for many of the genes that we identify as positively selected, it is nonetheless important to realize that positive selection of these genes indicates such loci have important functions, which in many instances may be integral to the unique adaptive features of each lineage. Several recent studies have harnessed the power of modern molecular selection analyses to direct functional experimentation based on the resulting molecular evolutionary hypotheses (see for example, [52-54]). It is our hope and intention that the identification and cataloging of these loci (Additional data file 5) for this and other groups of bacteria will serve as an evolutionary shortcut for others to design laboratory mutation experiments to assess the specific functional significance of these genes.

\section{Materials and methods Ortholog retrieval}

Twenty six genomes of Streptococcus were downloaded from GenBank (Table 1), representing six different species. Coding sequences were extracted from GenBank files, and orthologs were determined using OrthoMCL [43]. This program first makes an all-against-all BLASTp, and then defines putative pairs of orthologs or recent paralogs based on reciprocal BLAST. Recent paralogs are identified as genes within the same genome that are reciprocally more similar to each other than any sequence from another genome. OrthoMCL then converts the reciprocal BLAST $p$ values to a normalized similarity matrix that is analyzed by a Markov Cluster algorithm (MCL) [55]. In return, the MCL yields a set of clusters, with each cluster containing a set of orthologs and/or recent paralogs. OrthoMCL was run with a BLAST E-value cut-off of $1 \mathrm{e}-$ 5 , and an inflation parameter of 1.5. We used the OrthoMCL output to construct a table describing genome gene content (Additional data file 1). Genes that were not included in a cluster were considered taxon specific genes only if they were at least 50 amino acids long and had no BLAST hit with any other protein (E-value $\leq 1 \mathrm{e}-10)$. Preliminary analysis indicated that many truncated proteins found at the ends of contigs of the incomplete genomes, although exhibiting clear evidence of homology, were not included in any cluster because they had weak or no reciprocal BLAST hit. This table was used to plot venn diagrams with R 2.2.1 [56] and to construct four core-genome data-sets corresponding to the following taxa: genus Streptococcus, S. agalactiae, S. pyogenes, and $S$. thermophilus.

Gene loss, acquisition, and duplication were determined on all branches of trees involving these taxa using the parsimony criterion with the DelTran option, implemented in Paup 4.ob10 [57]. Ancestral gene state reconstruction was run with two different step-matrices: the first one assesses gene gain, loss and duplication as being equally likely (for example, [23]), while the second penalizes gene gain by assuming a double cost compared to loss and duplication (for example, [24]). The gene content table was also used to perform gene accumulation curves using $\mathrm{R}$, which describe the number of new genes and genes in common, with the addition of new comparative genomes. The procedure was repeated 100 times by randomly modifying genome insertion order to obtain means and standard errors.

\section{Alignments}

Orthologs were first aligned at the DNA level with ClustalW 1.82 [58]. To ensure homology, alignments that contained less than 35\% conserved sites for the Streptococcus genus data set, and 50\% for the Streptococcus species data sets, were discarded. A preliminary analysis of the data revealed that many sequences identified as under positive selection contained frameshifts that disrupted the reading frame (a single insertion or deletion that modified the reading frame), resulting in high non-synonymous substitution rates. Unfortunately, it is not possible to accurately discriminate sequencing errors from actual insertions or deletions; however, most of these frameshifts were found within the unclosed genomes and appeared at the beginning or end of the contigs, where sequencing errors are more probable. As described by Perrodou et al. [59], most of these frameshifts are probably sequencing errors, although it is possible that some are not [6o]. We chose the conservative approach of removing all codons appearing before or after the frameshift when located at the beginning or end of the coding sequence, respectively. For that purpose, Perl scripts were developed to find frameshifts on the DNA alignments, and the sequences were edited manually. A second alignment step was then used to refine all alignments by translating sequences to amino acids, aligning them with ClustalW, and then back-translating to DNA, using the script transAlign [61]. Finally, amino acid alignments showing a low percentage of conserved sites were manually inspected, and removed if the alignment was ambiguous.

\section{Recombination detection}

Both phylogenetic and substitution pattern methods were use to detect recombination events. The phylogenetic methods rely on the examination of phylogenetic congruence among genes (for example, [62,63]). If a gene has been laterally 
transferred, the phylogenetic relationships depicted by this gene will be different from the species tree. We first applied the method suggested by Susko et al. [34], which tests for the rejection of a set of topologies by a set of orthologous genes using the AU test [64]. When possible (that is, the number of taxa $\leq 7)$, the trees tested are all the possible unrooted trees (for example, [65]). When a gene rejects a tree that is supported by the majority of the other genes, this gene is considered to have been laterally transferred. We applied this approach to our data sets (except for $S$. thermophilus, which contains only three taxa), by using as tested topologies the individual gene trees obtained by phyML (general time reversible (GTR) $+\Gamma 4+\mathrm{I}$ model of evolution with a BIONJ starting tree) [66], with the addition of the tree obtained with Paup (GTR+Г4+I model of evolution, neighbor-joining (NJ) starting tree, and a tree-bisection-reconnection (TBR) branch-swapping algorithm) reconstructed from the concatenation of all genes. The site likelihood of each tree was than computed by the program baseml (PAML package) [67] using a GTR $+\Gamma 4$ model of evolution. The AU test was then applied using Consel [68].

As suggested by Susko et al. [34], results ( $p$ values for the rejection of each tree) were plotted using heatmaps obtained with $\mathrm{R}$. This approach has the disadvantage of been developed with a test (the AU test) that assesses the rejection of a tree by a gene and not for its acceptance [34]. As a result it is not possible to say if a tree is not rejected because it is not significantly different or because it is simply unresolved. This sort of situation is particularly expected with weakly divergent alignments, and at the opposite spectrum, with saturated alignments. We thus developed and performed a second set of analyses to complement the Susko approach and intended to quantify the amount of supported and incongruent phylogenetic signal between two gene trees. This approach relies on the discovery of well supported conflicting bipartitions (that is, branches that can not be observed in the same tree), as measured by non-parametric bootstrap analysis [69], thus revealing incongruence between gene histories. Support for each bipartition was obtained by bootstrapping a maximum likelihood (ML) tree search using Paup (GTR $+\Gamma 4+$ I model of evolution). Custom-made scripts were then used to find and count well supported ( $\geq 90 \%$ bootstrap support) conflicting bipartitions between gene trees. Additional to phylogenetic recombination detection, we employed methods specifically developed to detect homologous intragenic recombination. We used the compatibility approach between site histories, based on the pairwise homoplasy index (PHI), developed by Bruen et al. [70] and implemented within the program PhiPack [71]. Bruen et al. have suggested $\mathrm{PHI}$ is a more robust and sensitive method than many of the earlier approaches. Additional to the PHI statistic, for comparative purposes, we computed MaxChi [72] and NSS [73]p values for recombination using PhiPack (employing 1,000 permutations).

\section{Positive selection analysis}

We employed the branch-site test of Yang and Nielsen [20,74] implemented in the program Codeml of the package PAML [67] to assess positive selection at particular sites and lineages. Briefly, the likelihood of a model that does not allow positive selection is compared to one allowing positive selection on some specified lineages. The model allowing positive selection is tested using a likelihood ratio test (LRT) that is compared to a $\chi^{2}$ statistic with two degrees of freedom. Likelihoods were estimated on the genes or species trees. For the Streptococcus data set each lineage leading to the six different species was tested. For the species analyses each of the lineages corresponding with the different strains was tested, as well as several internal branches supported by the majority of genes. Finally, $p$ values were corrected for multiple hypothesis testing using the Benjamini and Yekutieli method [75]. The effect of lineages, and genes' TIGR main role categories, and their interaction were tested using an analysis of variance on the LRT, using R. Role categories containing less than ten genes were merged. If F-statistic was significant, Tukey's 'honest significant difference' multi-comparison method was used to discriminate lineages and role categories associated with different LRTs.

\section{Additional data files}

The following data are available with the online version of this paper. Additional data file 1 is a gene content table that describes the presence and absence of gene clusters per genome. Additional data file 2 is a CSV text file listing the composition of the clusters, with links to GenBank protein accession numbers. Additional data file 3 contains several tables detailing the gene gain, loss and duplication parsimony reconstruction for the Streptococcus, S. pyogenes and S. agalactiae pan-genomes. Results are presented for equal weights as well as following a model that penalized gene gain with respect to gene loss. Additional data file 4 is a table listing the genes showing evidence of recombination and positive selection. Additional data file 5 is a table listing the genes and lineages under positive selection for the four analyzed coregenomes, with gene annotation from NCBI and TIGR.

\section{Acknowledgements}

We thank Qi Sun and Robert Bukowsky for their help with the parallelization of the analyses on a Windows cluster at the Computational Biology Service Unit of Cornell University, Adam Siepel for helpful comments regarding the frameshift problem, and Paulina Pavinski Bitar for help regarding the manual frameshift check. This work was supported by Cornell University start-up funds and USDA grant 2006-35204-17422, awarded to MJS.

\section{References}

I. Awadalla P: The evolutionary genomics of pathogen recombination. Nat Rev Genet 2003, 4:50-60.

2. Feil EJ, Holmes EC, Bessen DE, Chan MS, Day NP, Enright MC, Goldstein R, Hood DW, Kalia A, Moore CE, et al.: Recombination within natural populations of pathogenic bacteria: shortterm empirical estimates and long-term phylogenetic consequences. Proc Natl Acad Sci USA 2001, 98: I82-I87. 
3. Spratt BG, Hanage WP, Feil E): The relative contributions of recombination and point mutation to the diversification of bacterial clones. Curr Opin Microbiol 200I, 4:602-606.

4. Hao W, Golding GB: The fate of laterally transferred genes: life in the fast lane to adaptation or death. Genome Res 2006, 16:636-643.

5. Ochman $H$, Lerat $E$, Daubin $V$ : Examining bacterial species under the specter of gene transfer and exchange. Proc Natl Acad Sci USA 2005, I 02(SuppI I):6595-6599.

6. Charlesworth J, Eyre-Walker A: The rate of adaptive evolution in enteric bacteria. Mol Biol Evol 2006, 23:1348-1356.

7. Chen SL, Hung CS, Xu J, Reigstad CS, Magrini V, Sabo A, Blasiar D, Bieri T, Meyer RR, Ozersky P, et al.: Identification of genes subject to positive selection in uropathogenic strains of Escherichia coli: a comparative genomics approach. Proc Natl Acad Sci USA 2006, 103:5977-5982.

8. Snider LA, Swedo SE: PANDAS: current status and directions for research. Mol Psychiatry 2004, 9:900-907.

9. Glaser P, Rusniok C, Buchrieser C, Chevalier F, Frangeul L, Msadek T, Zouine M, Couve E, Lalioui L, Poyart C, et al.: Genome sequence of Streptococcus agalactiae, a pathogen causing invasive neonatal disease. Mol Microbiol 2002, 45: I499-I5I3.

10. Farley MM: Group B streptococcal disease in nonpregnant adults. Clin Infect Dis 200I, 33:556-56I.

11. Lancefield RC: A serological differenciation of specific types of bovine hemolytic streptococci (group B). J Exp Med 1934, 59:44I-458

12. O'Brien KL, Nohynek H: Report from a WHO Working Group: standard method for detecting upper respiratory carriage of Streptococcus pneumoniae. Pediatr Infect Dis J 2003, 22: I-II.

13. Loesche WJ: Role of Streptococcus mutans in human dental decay. Microbiol Rev 1986, 50:353-380.

14. Smith HE, Buijs H, Wisselink HJ, Stockhofe-Zurwieden N, Smits MA: Selection of virulence-associated determinants of Streptococcus suis serotype 2 by in vivo. Infect Immun 200I, 69:1961-1966.

15. Tang J, Wang C, Feng Y, Yang W, Song H, Chen Z, Yu H, Pan X, Zhou $X$, Wang $H$, et al: Streptococcal toxic shock syndrome caused by Streptococcus suis serotype 2. PLoS Med 2006, 3: I I I.

16. Marri P, Hao W, Golding G: Gene gain and gene loss in Streptococcus: is it driven by habitat? Mol Biol Evol 2006, 23:2379-2391.

17. Nielsen R, Yang Z: Likelihood models for detecting positively selected amino acid sites and applications to the HIV-I envelope gene. Genetics 1998, 148:929-936.

18. Yang Z, Nielsen R: Estimating synonymous and nonsynonymous substitution rates under realistic evolutionary models. Mol Biol Evol 2000, 17:32-43.

19. Yang Z, Nielsen R, Goldman N, Pedersen AM: Codon-substitution models for heterogeneous selection pressure at amino acid sites. Genetics 2000, I 55:43 I-449.

20. Yang Z, Nielsen R: Codon-substitution models for detecting molecular adaptation at individual sites along specific lineages. Mol Biol Evol 2002, 19:908-917.

21. Yang Z, Wong WSW, Nielsen R: Bayes empirical bayes inference of amino acid sites under positive selection. Mol Biol Evol 2005, 22:1107-III8.

22. Tettelin H, Masignani V, Cieslewicz MJ, Donati C, Medini D, Ward NL, Angiuoli SV, Crabtree J, Jones AL, Durkin AS, et al.: Genome analysis of multiple pathogenic isolates of Streptococcus agalactiae: implications for the microbial "pan-genome". Proc Natl Acad Sci USA 2005, 102: 13950 - 13955.

23. Hao W, Golding GB: Patterns of bacterial gene movement. Mol Biol Evol 2004, 21 : 1 294- 1307.

24. Makarova K, Slesarev A, Wolf Y, Sorokin A, Mirkin B, Koonin E, Pavlov A, Pavlova N, Karamychev V, Polouchine N, et al.: Comparative genomics of the lactic acid bacteria. Proc Natl Acad Sci USA 2006, 103:15611-15616.

25. Anisimova M, Bielawski J, Yang Z: Accuracy and power of Bayes prediction of amino acid sites under positive selection. Mo Biol Evol 2002, 19:950-958.

26. Boussau B, Karlberg EO, Frank AC, Legault BA, Andersson SGE: Computational inference of scenarios for alpha-proteobacterial genome evolution. Proc Natl Acad Sci USA 2004, I01:9722-9727.

27. Snel B, Bork P, Huynen MA: Genomes in flux: the evolution of archaeal and proteobacterial gene content. Genome Res 2002, 12:17-25.

28. Kunin $\mathrm{V}$, Ouzounis $C A$ : The balance of driving forces during genome evolution in prokaryotes. Genome Res 2003, 13:1589-1594.

29. Csurös M, Miklós I: A probabilistic model for gene content evolution with duplication, loss and horizontal transfer. In Research in Computational Molecular Biology Edited by: Apostolico A, Guerra C, Istrail S, Pevzner P, Waterman M. Berlin: Springer; 2006:206-220. [Kanade T, Kittler ], Kleinberg JM, Mattern F, Mitchell JC, Nierstrasz O, Noar M, Rangan CP, Steffen B, Sudan M, et al. (Series Editors): Lecture Notes in Computer Science, vol. 3909]

30. Brochet M, Couvé E, Zouine M, Vallaeys T, Rusniok C, Lamy MC, Buchrieser C, Trieu-Cuot P, Kunst F, Poyart C, et al.: Genomic diversity and evolution within the species Streptococcus agalactiae. Microbes Infect 2006, 8: I227-I243.

31. Daubin V, Moran NA, Ochman H: Phylogenetics and the cohesion of bacterial genomes. Science 2003, 30 I:829-832.

32. Lerat E, Daubin V, Moran NA: From gene trees to organismal phylogeny in prokaryotes: the case of the gamma-Proteobacteria. PLOS Biol 2003, I:EI9.

33. Lerat E, Daubin V, Ochman H, Moran NA: Evolutionary origins of genomic repertoires in bacteria. PLOS Biol 2005, 3: el 30.

34. Susko E, Leigh J, Doolittle WF, Bapteste E: Visualizing and assessing phylogenetic congruence of core gene sets: a case study of the gamma-proteobacteria. Mol Biol Evol 2006, 23:1019-1030.

35. Täpp J, Thollesson M, Herrmann B: Phylogenetic relationships and genotyping of the genus Streptococcus by sequence determination of the RNase P RNA gene, rnpB. Int J Syst Evol Microbiol 2003, 53:186I-I87|

36. Arends JP, Zanen HC: Meningitis caused by Streptococcus suis in humans. Rev Infect Dis 1988, 10:131-137.

37. Anisimova M, Nielsen R, Yang Z: Effect of recombination on the accuracy of the likelihood method for detecting positive selection at amino acid sites. Genetics 2003, 164:1229-1236.

38. Davies HD, McGeer A, Schwartz B, Green K, Cann D, Simor AE, Low DE: Invasive group A streptococcal infections in Ontario, Canada. Ontario Group A Streptococcal Study Group. N EnglJ Med 1996, 335:547-554.

39. Kaul R, McGeer A, Low DE, Green K, Schwartz B: Populationbased surveillance for group $A$ streptococcal necrotizing fasciitis: Clinical features, prognostic indicators, and microbiologic analysis of seventy-seven cases. Ontario Group A Streptococcal Study. Am J Med 1997, 103: 18-24.

40. Li Z, Sakota V, jackson D, Franklin AR, Beall B: Array of M protein gene subtypes in 1064 recent invasive group A streptococcus isolates recovered from the active bacterial core surveillance. J Infect Dis 2003, I 88: I587-1592.

4I. O'Brien KL, Beall B, Barrett NL, Cieslak PR, Reingold A, Farley MM, Danila R, Zell ER, Facklam R, Schwartz B, et al.: Epidemiology of invasive group A Streptococcus disease in the United States, 1995-1999. Clin Infect Dis 2002, 35:268-276.

42. Sharkawy A, Low DE, Saginur R, Gregson D, Schwartz B, Jessamine $P$, Green K, McGeer A: Severe group a streptococcal soft-tissue infections in Ontario: 1992-1996. Clin Infect Dis 2002, 34:454-460.

43. Li L, Stoeckert CJJ, Roos DS: OrthoMCL: identification of ortholog groups for eukaryotic genomes. Genome Res 2003, 13:2178-2|89.

44. Paik S, Senty L, Das S, Noe JC, Munro CL, Kitten T: Identification of virulence determinants for endocarditis in Streptococcus sanguinis by signature-tagged mutagenesis. Infect Immun 2005, 73:6064-6074

45. Mengin-Lecreulx D, Falla T, Blanot D, van Heijenoort J, Adams DJ, Chopra I: Expression of the Staphylococcus aureus UDP-N. acetylmuramoyl-L-alanyl-D-glutamate:L-lysine ligase in Escherichia coli and effects on peptidoglycan biosynthesis and cell growth. J Bacteriol 1999, 181:5909-5914.

46. Beres SB, Richter EW, Nagiec MJ, Sumby P, Porcella SF, DeLeo FR, Musser JM: Molecular genetic anatomy of inter- and intraserotype variation in the human bacterial pathogen group $A$ Streptococcus. Proc Natl Acad Sci USA 2006, 103:7059-7064.

47. Luan SL, Granlund M, Sellin M, Lagergard T, Spratt BG, Norgren M: Multilocus sequence typing of Swedish invasive group $B$ streptococcus isolates indicates a neonatally associated genetic lineage and capsule switching. J Clin Microbiol 2005, 43:3727-3733.

48. Bisharat N, Crook DW, Leigh J, Harding RM, Ward PN, Coffey T], Maiden MC, Peto T, Jones N: Hyperinvasive neonatal group B streptococcus has arisen from a bovine ancestor. J Clin Microbiol $2004,42: 216 \mid-2167$ 
49. Kim YR, Brinsmade SR, Yang Z, Escalante-Semerena J, Fierer J: Mutation of phosphotransacetylase but not isocitrate lyase reduces the virulence of Salmonella enterica serovar Typhimurium in mice. Infect Immun 2006, 74:2498-2502.

50. Lindahl G, Stalhammar-Carlemalm M, Areschoug T: Surface proteins of Streptococcus agalactiae and related proteins in other bacterial pathogens. Clin Microbiol Rev 2005, 1 8:102-127.

5I. Piddock LJV: Multidrug-resistance efflux pumps - not just for resistance. Nat Rev Microbiol 2006, 4:629-636.

52. Bielawski JP, Dunn KA, Sabehi G, Beja O: Darwinian adaptation of proteorhodopsin to different light intensities in the marine environment. Proc Natl Acad Sci USA 2004, I 0 I: I 4824- I 4829.

53. Ivarsson Y, Mackey AJ, Edalat M, Pearson WR, Mannervik B: Identification of residues in glutathione transferase capable of driving functional diversification in evolution. A novel approach to protein redesign. J Biol Chem 2003, 278:8733-8738.

54. Sawyer SL, Wu LI, Emerman M, Malik HS: Positive selection of primate TRIM5alpha identifies a critical species-specific retroviral restriction domain. Proc Natl Acad Sci USA 2005, 102:2832-2837.

55. VanDongen S: Graph clustering by flow simulation. In $P h D$ thesis University of Utrecht, The Netherlands; 2000.

56. The R Project for Statistical Computing [http://www.rproject.org/]

57. Swofford DL: PAUP*. Phylogenetic Analysis Using Parsimony (*and Other Methods). Version 4 Sunderland, Massachusetts: Sinauer Associates; 2002.

58. Thompson J, Higgins D, Gibson T: CLUSTAL W: improving the sensitivity of progressive multiple sequence alignment through sequence weighting, positions-specific gap penalties and weight matrix choice. Nucleic Acids Res Suppl 1994, 22:4673-4680.

59. Perrodou E, Deshayes C, Muller J, Schaeffer C, VanDorsselaer A, Ripp $R$, Poch O, Reyrat JM, Lecompte O: ICDS database: interrupted CoDing sequences in prokaryotic genomes. Nucleic Acids Res Suppl 2006, 34(Database issue):D338-D343.

60. Medigue $C$, Rose M, Viari A, Danchin A: Detecting and analyzing DNA sequencing errors: toward a higher quality of the Bacillus subtilis genome sequence. Genome Res 1999, 9: I I 16- I I 27.

61. Bininda-Emonds ORP: transAlign: using amino acids to facilitate the multiple alignment of protein-coding DNA sequences. BMC Bioinformatics 2005, 6:156.

62. Holmes EC, Urwin R, Maiden MC: The influence of recombination on the population structure and evolution of the human pathogen Neisseria meningitidis. Mol Biol Evol 1999, 16:741-749.

63. Feil EJ, Holmes EC, Bessen DE, Chan MS, Day NP, Enright MC, Goldstein R, Hood DW, Kalia A, Moore CE, et al.: Recombination within natural populations of pathogenic bacteria: shortterm empirical estimates and long-term phylogenetic consequences. Proc Natl Acad Sci USA 2001, 98:182-187.

64. Shimodaira $\mathrm{H}$ : An approximately unbiased test of phylogenetic tree selection. Syst Biol 2002, $51: 492-508$.

65. Filée J, Bapteste E, Susko E, Krisch HM: A selective barrier to horizontal gene transfer in the T4-type bacteriophages that has preserved a core genome with the viral replication and structural genes. Mol Biol Evol 2006, 23:1688-1696.

66. Guindon S, Gascuel O: A simple, fast, and accurate algorithm to estimate large phylogenies by maximum likelihood. Syst Biol 2003, 52:696-704.

67. Yang Z: PAML: a program package for phylogenetic analysis by maximum likelihood. Comput Appl Biosci 1997, 1 3:555-556.

68. Shimodaira $H$, Hasegawa M: CONSEL: for assessing the confidence of phylogenetic tree selection. Bioinformatics 200I, 17:1246-1247.

69. Felsenstein J: Confidence limits on phylogenies: An approach using the bootstrap. Evolution Int J Org Evolution 1985, 39:783-791.

70. Bruen TC, Philippe $H$, Bryant D: A simple and robust statistical test for detecting the presence of recombination. Genetics 2006, I 172:2665-268।.

7I. Trevor's Bruen homepage [http://www.mcb.mcgill.ca/ trevor/]

72. MaynardSmith J: Analyzing the mosaic structure of genes. J Mol Evol 1992, 34:126-129.

73. Jakobsen IB, Easteal S: A program for calculating and displaying compatibility matrices as an aid in determining reticulate evolution in molecular sequences. Comput Appl Biosci 1996, | 2:29|-295.

74. Zhang J, Nielsen R, Yang Z: Evaluation of an improved branchsite likelihood method for detecting positive selection at the molecular level. Mol Biol Evol 2005, 22:2472-2479.

75. Benjamini $Y$, Yekutieli D: The control of the false discovery rate in multiple testing under dependency. Ann Stat 200I, 29: I 165- II88.

76. Ferretti JJ, McShan WM, Ajdic D, Savic DJ, Savic G, Lyon K, Primeaux C, Sezate S, Suvorov AN, Kenton S, et al.: Complete genome sequence of an MI strain of Streptococcus pyogenes. Proc Natl Acad Sci USA 200 I, 98:4658-4663.

77. Sumby P, Porcella SF, Madrigal AG, Barbian KD, Virtaneva K, Ricklefs SM, Sturdevant DE, Graham MR, Vuopio-Varkila J, Hoe NP, et al: Evolutionary origin and emergence of a highly successful clone of serotype MI group a Streptococcus involved multiple horizontal gene transfer events. J Infect Dis 2005, 192:77I-782.

78. Smoot JC, Barbian KD, VanGompel JJ, Smoot LM, Chaussee MS, Sylva GL, Sturdevant DE, Ricklefs SM, Porcella SF, Parkins LD, et al: Genome sequence and comparative microarray analysis of serotype MI 8 group A Streptococcus strains associated with acute rheumatic fever outbreaks. Proc Natl Acad Sci USA 2002 99:4668-4673.

79. Beres SB, Sylva GL, Barbian KD, Lei B, Hoff JS, Mammarella ND, Liu MY, Smoot JC, Porcella SF, Parkins LD, et al.: Genome sequence of a serotype M3 strain of group A Streptococcus: phageencoded toxins, the high-virulence phenotype, and clone emergence. Proc Natl Acad Sci USA 2002, 99: 10078-10083.

80. Green NM, Zhang S, Porcella SF, Nagiec MJ, Barbian KD, Beres SB, LeFebvre RB, Musser JM: Genome sequence of a serotype M28 strain of group a Streptococcus: potential new insights into puerperal sepsis and bacterial disease specificity. J Infect Dis 2005, 192:760-770.

8I. Nakagawa I, Kurokawa K, Yamashita A, Nakata M, Tomiyasu Y, Okahashi N, Kawabata S, Yamazaki K, Shiba T, Yasunaga T, et al.: Genome sequence of an M3 strain of Streptococcus pyogenes reveals a large-scale genomic rearrangement in invasive strains and new insights into phage evolution. Genome Res 2003, I 3:1042-1055.

82. Banks DJ, Porcella SF, Barbian KD, Beres SB, Philips LE, Voyich JM, DeLeo FR, Martin JM, Somerville GA, Musser JM: Progress toward characterization of the group A Streptococcus metagenome: complete genome sequence of a macrolide-resistant serotype M6 strain. J Infect Dis 2004, 190:727-738.

83. Hoskins J, Alborn WEJ, Arnold J, Blaszczak LC, Burgett S, DeHoff BS, Estrem ST, Fritz L, Fu DJ, Fuller W, et al.: Genome of the bacterium Streptococcus pneumoniae strain R6. J Bacteriol 200I, 183:5709-57|7

84. Tettelin H, Nelson KE, Paulsen IT, Eisen JA, Read TD, Peterson S, Heidelberg J, DeBoy RT, Haft DH, Dodson RJ, et al:: Complete genome sequence of a virulent isolate of Streptococcus pneumoniae. Science 2001, 293:498-506.

85. Ajdic D, McShan WM, McLaughlin RE, Savic G, Chang J, Carson MB, Primeaux C, Tian R, Kenton S, jia $\mathrm{H}$, et al.: Genome sequence of Streptococcus mutans UAI59, a cariogenic dental pathogen. Proc Natl Acad Sci USA 2002, 99: I 4434-I 4439.

86. Tettelin H, Masignani V, Cieslewicz MJ, Eisen JA, Peterson S, Wessels MR, Paulsen IT, Nelson KE, Margarit I, Read TD, et al.: Complete genome sequence and comparative genomic analysis of an emerging human pathogen, serotype V Streptococcus agalactiae. Proc Natl Acad Sci USA 2002, 99: I239|-12396.

87. Bolotin A, Quinquis B, Renault P, Sorokin A, Ehrlich SD, Kulakauskas S, Lapidus A, Goltsman E, Mazur M, Pusch GD, et al.: Complete sequence and comparative genome analysis of the dairy bacterium Streptococcus thermophilus. Nat Biotechnol 2004, 22: $1554-1558$. 